\title{
Growth and Switching of Ferroelectric Nanocrystals from Ultrathin Film of Copolymer of Vinylidene Fluoride and Trifluoroethylene
}

\author{
R. Gaynutdinov, ${ }^{1}$ V. Fridkin, ${ }^{1}$ and H. Kliem $^{2}$ \\ ${ }^{1}$ Institute of Crystallography, Russian Academy of Sciences, Moscow, 119333, Russia \\ ${ }^{2}$ Institute of Electrical Engineering Physics, Saarland University, 66041, Saarbrücken, Germany \\ Correspondence should be addressed to V. Fridkin, fridkin@ns.crys.ras.ru
}

Received 16 February 2011; Accepted 6 May 2011

Academic Editor: Marisol Reyes-Reyes

Copyright ( 2011 R. Gaynutdinov et al. This is an open access article distributed under the Creative Commons Attribution License, which permits unrestricted use, distribution, and reproduction in any medium, provided the original work is properly cited.

\begin{abstract}
The ferroelectric nanocrystals of the copolymer of vinylidene fluoride and trifluoroethylene P(VDF-TrFE) were grown from ultrathin Langmuir-Blodgett (LB) films on Si substrate. The annealing of ultrathin LB films with thickness of 3 monolayers $(5 \mathrm{~nm})$ in air in paraelectric phase at temperature $125^{\circ} \mathrm{C}$ was performed. The self-assembly leads to the growth of nanocrystals of ferroelectric copolymer 15-25 nm thick and 100-200 $\mathrm{nm}$ in diameter. The nanocrystals presumably belong to orthorhombic space group, where axis 2 is the direction of spontaneous polarization (and normal to substrate). By means of atomic force microscopy (AFM), the kinetics of ferroelectric nanocrystals growth and their switching were investigated. The obtained results confirm the conclusions that copolymer nanocrystals are candidates for high-density nonvolatile storage media devices.
\end{abstract}

\section{Introduction}

The first studies of independent nanocrystals of the copolymer of vinylidene fluoride and trifluoroethylene P(VDFTrFE) were reported in $[1,2]$. They were prepared from ultrathin LB films ( $1-5$ monolayers) by annealing in air in the paraelectric phase at temperature $125^{\circ} \mathrm{C}$ with heating and cooling rates of $1^{\circ} \mathrm{C}$ per min. The ultrathin films were prepared by horizontal (Schaefer) LB method on Si substrate, investigated and described in detail in [3-5]. As shown in $[1,2]$, self-assembly leads to growth of nanocrystals of ferroelectric copolymer with thickness approximately $15-25 \mathrm{~nm}$ and diameter approximately $100-200 \mathrm{~nm}$. These dimensions depend on the composition of copolymer, number of monolayers, and the annealing temperature.

Ferroelectricity in copolymer nanocrystals was confirmed by polarization reversal evident in the hysteresis of both capacitance and pyroelectric response of arrays of nanocrystals [1]. The switching time of nanocrystals arrays was measured by the pulsed current method indicating that they switch by nucleation and domain wall growth mechanisms [6]. In [7], by electron diffraction were obtained the lattice parameters of what appeared to be single lamellar nanocrystals, corresponded to the orthorhombic space group $\mathrm{mm} 2$, which is also consistent with ferroelectricity (axis 2 is special polar direction).

The first investigation of the ferroelectric properties of individual copolymer nanocrystals have been reported in $[8,9]$, where polarization switching and hysteresis loops were observed with the method of piezoresponse force microscopy. As a result, the domain mechanism of switching and existence of nanodomains in the copolymer nanocrystals were proposed. The kinetic studies of copolymer nanocrystals switching were also consistent with nucleation-limited switching dynamics $[6,9]$.

The polarization switching kinetics was reported recently [9] for individual nanocrystals of ferroelectric of copolymer approximately $15-25 \mathrm{~nm}$ thick and $100-200 \mathrm{~nm}$ in diameter. The nanocrystal polarization could be repeatedly switched between two stable states and exhibit a well-saturated hysteresis loop. The dependence of switching time on switching voltage for an individual nanocrystal thickness $h=25 \mathrm{~nm}$ follow, the Merz exponential form $\exp \left(E_{a} l / V\right)$ where the 


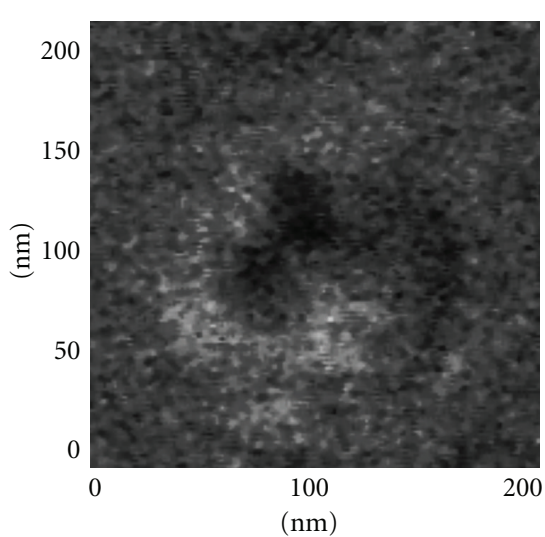

(a)

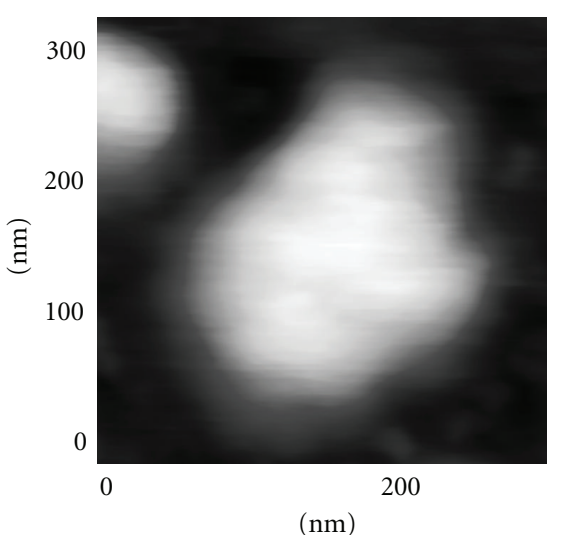

(d)

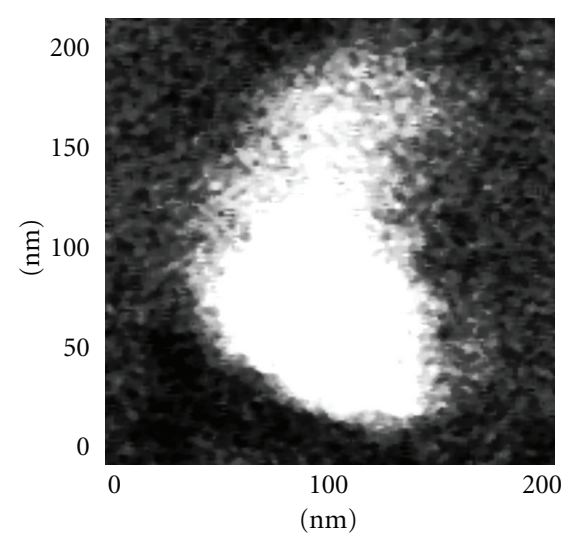

(b)

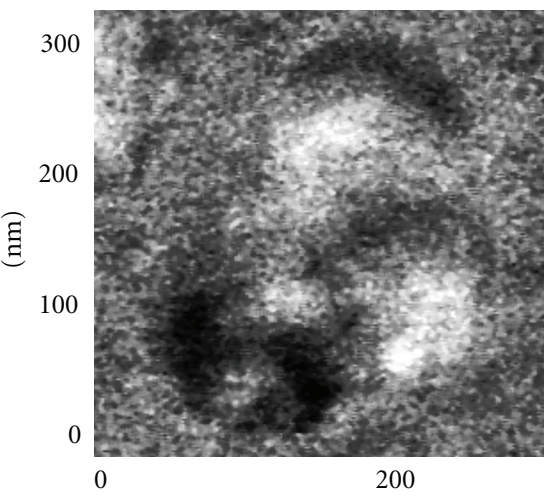

(nm)

(e)

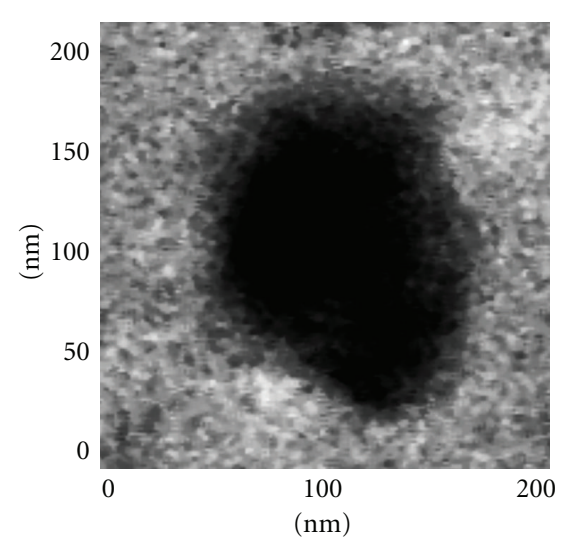

(c)

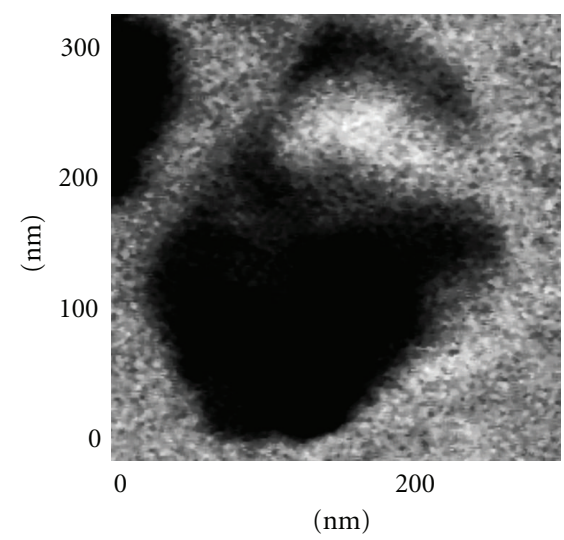

(f)

FIGURE 1: Piezoresponse images of the crystals at the following stages: nanocrystal no. $1(R \approx 50 \mathrm{~nm}, h \approx 15 \mathrm{~nm})$ (a) initial phase image, (b) after applying a bias voltage $+10 \mathrm{~V}$, and (c) after applying a bias voltage $-10 \mathrm{~V}$; nanocrystal no. $21(R \approx 100 \mathrm{~nm}, h \approx 25 \mathrm{~nm})(\mathrm{d})$ topography, (e) initial phase image, and (f) after applying a bias voltage $-10 \mathrm{~V}$.

activation field $E_{a}=1,4 \mathrm{GV} / \mathrm{m}$, a little larger than the value $1 \mathrm{GV} / \mathrm{m}$ obtained with thin-film capacitors of a similar $73: 27$ composition copolymer [10].

It was also found $[8,11]$ that the copolymer nanocrystals reveal multiple domains with opposing polarization states. What remains is to find out whether or not these crystals are homogeneous. The task of this paper is investigation of growth kinetics of the ferroelectric copolymer nanocrystals from the smallest size to the saturation, and observation of the nanocrystals switching in dependence of their size.

It is important to add, that in $[9,10]$ the kinetics of switching was performed for voltages $v>v_{c}$, where $v_{c}$ is the coercive voltage.

\section{The Fabrication and Observation of Nanocrystals}

The nanocrystals were fabricated from a ferroelectric copolymer by Langmuir-Blodgett deposition of a copolymer consisting of $70 \%$ vinylidene fluoride and $30 \%$ trifluoroethylene. Deposition was accomplished by dispersing a $1.3 \%$ by weight solution of the copolymer in dimethyl sulfoxide on the surface of ultrapure water at room temperature and compressed slowly to a pressure of $5 \mathrm{mN} / \mathrm{m}^{2}$. One monolayer was transferred to a silicon substrate coated with a $50 \mathrm{~nm}$ thick aluminum film. To make the nanocrystals the, film consisting from 3 monolayers was then heated to $125^{\circ} \mathrm{C}$ for different time and cooled to room temperature at a rate of $1^{\circ} \mathrm{C}$ per min. Measurements were carried out with a scanning probe microscope (model SOLVER P47, NTMDT, Moscow). The growth kinetics was investigated in tapping mode by means of silicon cantilevers (model TL01, MicroMasch, Estonia) which had a lever force constant of approximately $2 \mathrm{~N} / \mathrm{m}$, a resonant frequency of $90 \mathrm{kHz}$, and tip radius of approximately $10 \mathrm{~nm}$. The switching kinetics was studied with method of piezoresponse force microscopy (PFM). For the PFM measurements tips with $\mathrm{Ti} / \mathrm{Pt}$ conductive coating (CSC38, MicroMash, Estonia) were used. Tip radius is about $10 \mathrm{~nm}$, and lever force constant is approximately $0.05 \mathrm{~N} / \mathrm{m}$. The piezoresponse was obtained by applying an excitation voltage of amplitude $0.5 \mathrm{~V}$ and frequency $200 \mathrm{kHz}$ to the tip and recording the amplitude and phase of the resulting tip deflection signal from the cantilever position detector with a lock-in amplifier. The piezoresponse signal is enhanced, because the bias voltage frequency was just above the piezoresponse resonance frequency, which was between 180 and $190 \mathrm{kHz}$. The PFM images consist of $x-y$ 


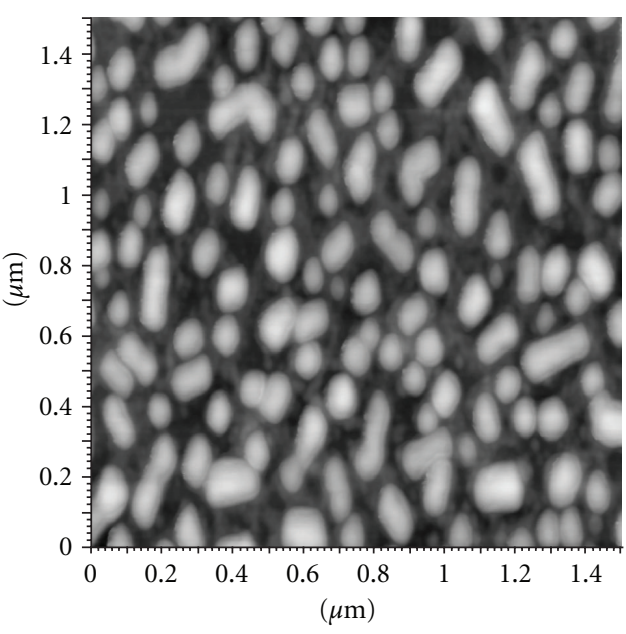

(a)

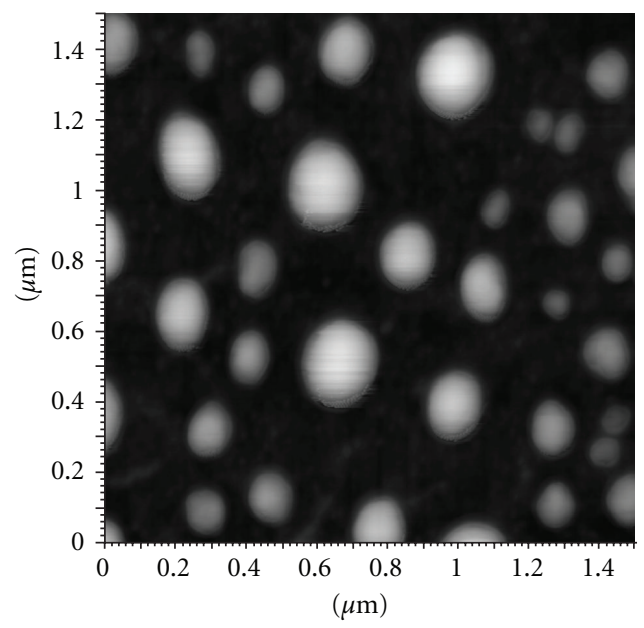

(c)

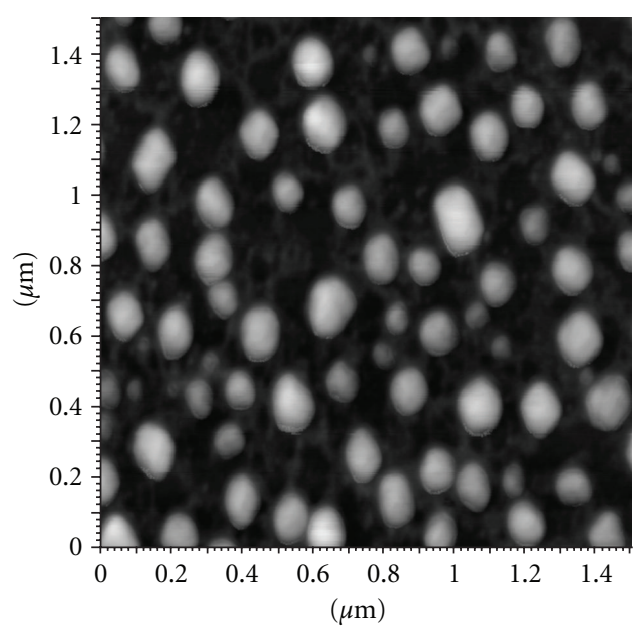

(b)

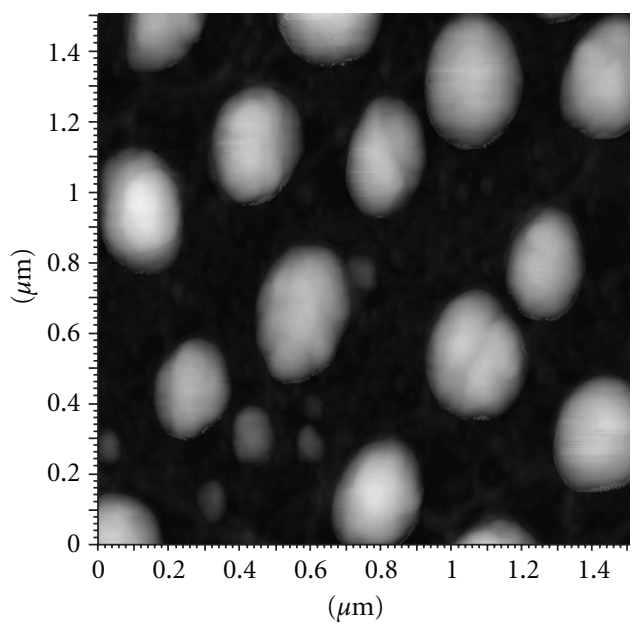

(d)

Figure 2: Kinetics of nanocrystals growth: (a) starting point, (b) $2 \mathrm{~min}$, (c) $3 \mathrm{~min}$, and (d) $4 \mathrm{~min}$.

maps of the composite PFM signal, which is equal to the PFM amplitude times the cosine of the PFM phase. The measurements were carried at room temperature. The selfassembly led to the creation of nanocrystals of ferroelectric copolymer approximately 15-25 nm thick and 50-100 nm in radius with somewhat irregular shapes.

\section{The Switching of Ferroelectric Copolymer Nanocrystals}

As we mentioned in introduction, the switching of copolymer nanocrystals, its kinetics, and hysteresis loops were investigated in $[6-9,11]$. In the present paper we revealed peculiarities in the crystal switching of different size.

Figure 1 shows the PFM image of small (Figures 1(a), $1(\mathrm{~b})$, and $1(\mathrm{c})$ ) and large (Figures 1(d), 1(e), and 1(f)) crystals, 1 and 2 . The images of crystal 1 are obtained for following three states: (a) initial phase image (b) after applying a bias voltage of $V=+10 \mathrm{~V}$, and (c) after applying a bias voltage of $V=-10 \mathrm{~V}$. For crystal 2 the following images are presented: (d) topography image; (e) initial phase image, and (f) after applying a bias voltage of $\mathrm{V}=-10 \mathrm{~V}$. The comparison of the PFM images for crystals 1 and 2 shows the homogeneous switching of the small crystal 1 and inhomogeneous switching of large crystal 2. The right upper side of crystal 2 is not switched and it does not depend on the position of the tip. At the same time the all small crystals reveal homogeneous switching. Perhaps it shows that some large crystals are not monocrystals, consisting of parts with different orientation. The other explanation is the existence of so-called "stubborn" domains, which are known in ferroelectric crystals. The solution could be found by the investigation of electron diffraction patterns and transmission electron microscopy images of the copolymer nanocrystals [7].

\section{Growth Kinetics}

The polymer film perturbation and nanocrystal formation kinetics were analyzed in detail in [12]. Here we consider the nanocrystals growth just after film perturbation with a voltage pulse applied to the AFM tip. 


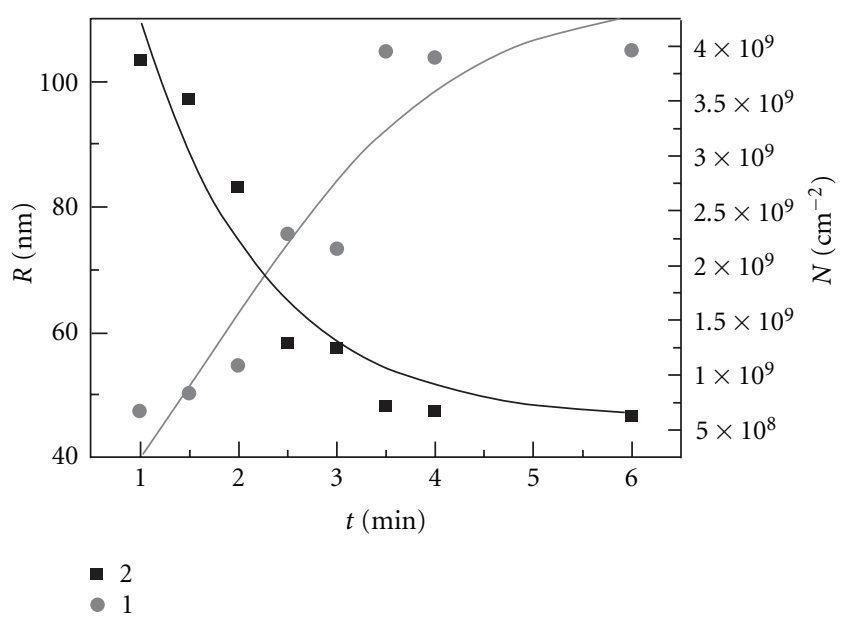

FIgURE 3: Time dependence of the middle radius $R$ and concentration $N$ (1: crystal concentration $N, 2$ : middle radius of crystals $R$ ).

Just after polymer film perturbation and the first smallest crystals formation, we keep them at temperature $+125^{\circ} \mathrm{C}$ for different periods of time ranging from $1 \mathrm{~min}$ to $6 \mathrm{~min}$. This leads to the growth of crystals and a decrease of their concentration. Figure 2 illustrates 4 AFM images, corresponding to the initial stage (just after the film perturbation) and to three different period of annealing.

Figure 3 shows the time dependence of the middle radius $R$ (curve 2, left side) and crystal concentration $N$ (curve 1, right side). The values $R$ and $N$ reveal saturation at approximately same relaxation time $\tau$. The experiment shows that the middle values of radius $R$ and height $h$ of the crystals grow from the initial values $R_{0} \approx 50 \mathrm{~nm}$ and $h_{0} \approx 15 \mathrm{~nm}$ to the saturation values $R_{s} \approx 100 \mathrm{~nm}$ and $h_{s} \approx 25 \mathrm{~nm}$.

This kinetics based on the well-known growth kinetics from the melt [13] could be explained by the simplest model of drops merging. At $T=125^{\circ} \mathrm{C}$ we can consider material as a plate of round drops with radius $R_{0}$, height $h_{0}$, and concentration $N_{0}$ (on the substrate surface). As the temperature decreases the drops are crystallized in the ferroelectric phase. Let us consider one of the drops. The flow of the other drops to it is given by $j_{+}$

$$
j_{+}=v \exp \left(-\frac{u_{1}}{k T}\right) N
$$

The flow of the material in the opposite direction is given by $j_{-}$

$$
j_{-}=v \exp \left(-\frac{u_{2}}{k T}\right) n,
$$

where $v, u_{1}$, and $u_{2}$ are frequency and energy of drops merging and tearing accordingly, $N$ and $n$ are concentration of drops outside of drop 1 and on its surface accordingly. The condition of growth of the drops leads to $u_{1}<u_{2}$. Then the time dependence of concentration $N$ is given by

$$
\frac{d N}{d t}=-j_{+}+j_{-}=-a_{1} N+a_{2} n, \quad N+n=N_{0},
$$

where $a_{1}=\nu \exp \left(-u_{1} / k T\right), a_{2}=\nu \exp \left(-u_{2} / k T\right), a_{2}<a_{1}$. The solution of ( 3 ) for $N(t=0)=N_{0}$ gives

$$
N=N_{0}\left\{\frac{a_{2}}{a_{1}+a_{2}}+\frac{a_{1}}{a_{1}+a_{2}} \exp \left(-\frac{t}{\tau}\right)\right\}, \quad \tau=\frac{1}{a_{1}+a_{2}} .
$$

The velocity of drop growth is given by

$$
\begin{aligned}
\frac{d R}{d t} & =\left(j_{+}-j_{-}\right) a S=\left\{a_{1} N-a_{2}\left(N_{0}-n\right)\right\} a S \\
& \cong a_{1} N a R^{2} \quad\left(a_{2} \ll a_{1}\right) .
\end{aligned}
$$

Here $a$ is lattice constant and $S$ is the length of the drop circle. The solution of (5) for $R(t=0)=R_{0}$ gives

$$
R=\frac{1}{\left(1 / R_{0}\right)-a_{1} a N_{0} \tau\{1-\exp (-t / \tau)\}},
$$

where $\tau$ is given by (4).

This kinetics model for the average size of the drops, neglecting the size distribution, explained the saturation of the concentration and size with the same relaxation time $\tau$. The relationship between saturated values of $N_{s}$ and $R_{s}$ is obtained from the conservation of copolymer mass:

$$
\frac{N_{s}}{N_{0}}\left(\frac{R_{s}}{R_{0}}\right)^{2} \frac{h_{s}}{h_{0}} \cong 1
$$

The substitution $N_{s} \approx 510^{8} \mathrm{sm}^{-2}, N_{0} \approx 410^{9} \mathrm{sm}^{-2}, R_{s} \approx$ $100 \mathrm{~nm}, R_{0} \approx 50 \mathrm{~nm}, h_{s} \approx 25 \mathrm{~nm}$, and $h_{0} \approx 15 \mathrm{~nm}$ meets (7).

\section{Conclusions}

The obtained results show the drastic difference in small and large nanocrystals switching. May be it is caused by heterogeneity of large copolymer nanocrystals. The size of copolymer nanocrystals at definite thickness of LB film is determined by the time and temperature of annealing.

The conditions of the ferroelectric nanocrystals growth and their switching are of critical importance to the performance of ferroelectric memory technology. Recently, the fabrication of high-density arrays of crystalline nanostructure of a ferroelectric copolymer $\mathrm{P}(\mathrm{VDF}-\mathrm{TrFE})$ was realized by nanoembossing [14]. The results of $[13,14]$ show that copolymer ferroelectric crystals are candidates for highdensity nonvolatile storage media devices.

\section{Acknowledgments}

The authors thank the late Professor Vitaly L. Ginzburg and Professor Stephen Ducharme for useful discussion. This paper was supported by Deutsche Forschung Gemeindschaft and by the Russian Research Foundation (RFFI-09-0200096).

\section{References}

[1] M. Bai and S. Ducharme, "Ferroelectric nanomesa formation from polymer Langmuir-Blodgett films," Applied Physics Letters, vol. 85, no. 16, pp. 3528-3530, 2004. 
[2] M. Bai, M. Poulsen, S. Ducharme, and M. Bai, "Effects of annealing conditions on ferroelectric nanomesa selfassembly," Journal of Physics Condensed Matter, vol. 18, no. 31, article 30, pp. 7383-7392, 2006.

[3] S. P. Palto, L. N. Blinov, A. Bune et al., "Ferroelectric Langmuir-Blodgett films," Ferroelectrics, Letters Section, vol. 19, no. 3-4, pp. 65-68, 1995.

[4] A. V. Bune, V. M. Fridkin, S. Ducharme et al., "Twodimensional ferroelectric films," Nature, vol. 391, no. 6670, pp. 874-877, 1998.

[5] S. Ducharme, S. P. Palto, V. M. Fridkin, and H. S. Nalwa, Eds., Handbook of Thin Materials, vol. 3, Academic Press, Moscow, Russia, 2002.

[6] C. M. Othon, J. Kim, S. Ducharme, and V. M. Fridkin, "Switching kinetics of ferroelectric polymer nanomesas," Journal of Applied Physics, vol. 104, no. 5, Article ID 054109, 2008.

[7] M. Bai, X. Z. Li, and S. Ducharme, "Electron diffraction study of the structure of vinylidene fluoride-trifluoroethylene copolymer nanocrystals," Journal of Physics Condensed Matter, vol. 19, no. 19, Article ID 196211, 2007.

[8] C. J. KIM, An intelligent decision making system for detecting high impedance faults, Ph.D. thesis, University of Nebraska, 2008.

[9] R. V. Gaynutdinov, O. A. Lysova, S. G. Yudin et al., "Polarization switching kinetics of ferroelectric nanomesas of vinylidene fluoride-trifluoroethylene copolymer," Applied Physics Letters, vol. 95, no. 2, Article ID 023303, 2009.

[10] T. Nakajima, R. Abe, Y. Takahashi, and T. Furukawa, "Intrinsic switching characteristics of ferroelectric ultrathin vinylidene fluoride/trifluoroethylene copolymer films revealed using Au electrode," Japanese Journal of Applied Physics, Part 2: Letters, vol. 44, no. 42-45, pp. L1385-L1388, 2005.

[11] C. Kittel, "Theory of the structure of ferromagnetic domains in films and small particles," Physical Review, vol. 70, no. 1112, pp. 965-971, 1946.

[12] P. Sharma, T. J. Reece, D. Wu et al., "Nanoscale domain patterns in ultrathin polymer ferroelectric films," Journal of Physics Condensed Matter, vol. 21, no. 48, Article ID 485902, 2009.

[13] A. Chernov, E. Givargizov, and Kh. Bagdasarov, Modern Crystallography, vol. 3, Springer, Berlin, Germany, 1980.

[14] J. Li, Y. Luo, M. Bai, and S. Ducharme, "A continuum model on the nanomesa and nanowell formation in Langmuir-Blodgett ferroelectric polymeric films," Journal of the Mechanics and Physics of Solids, vol. 54, no. 10, pp. 2162-2182, 2006. 

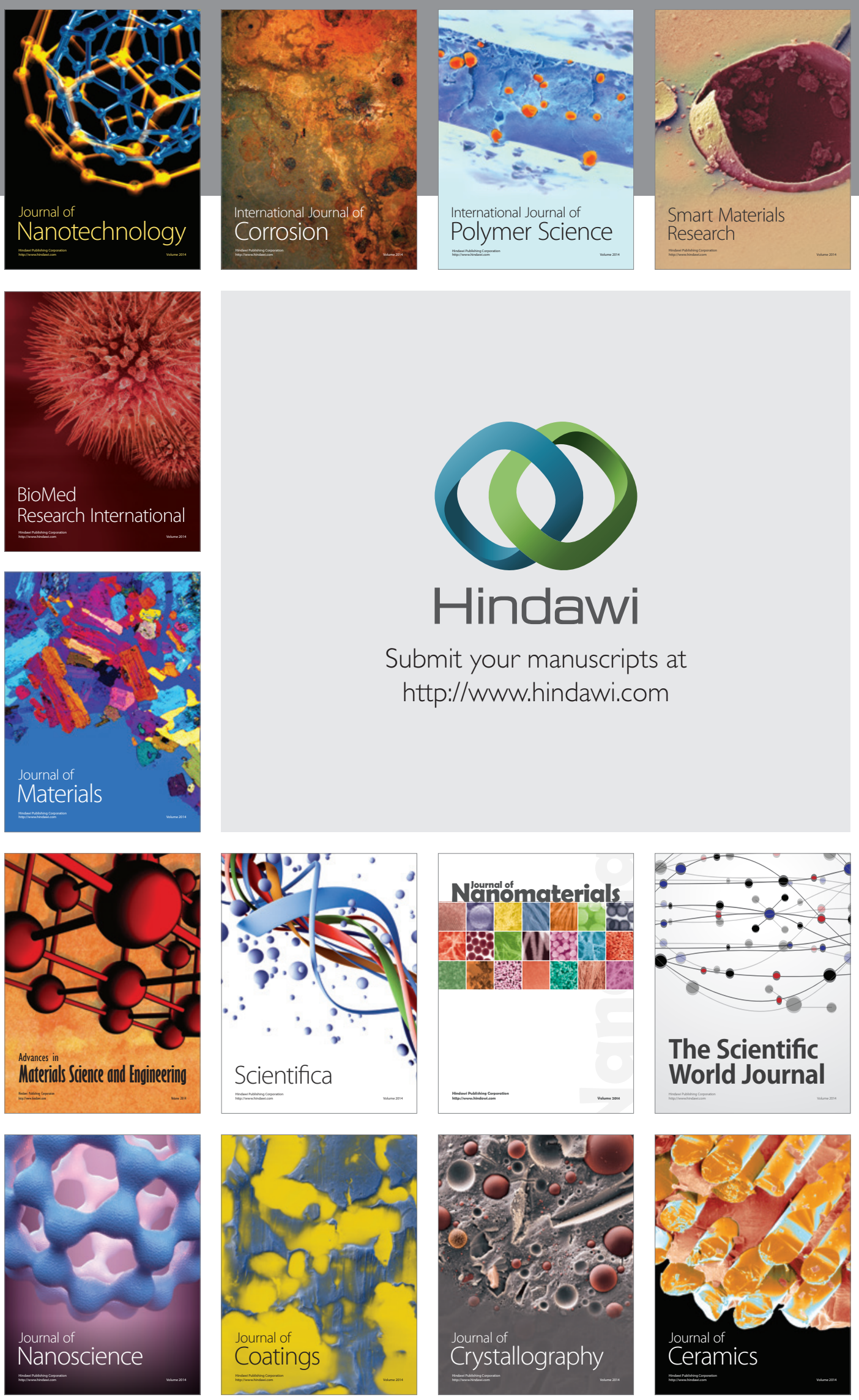

The Scientific World Journal

Submit your manuscripts at

http://www.hindawi.com

\section{World Journal}

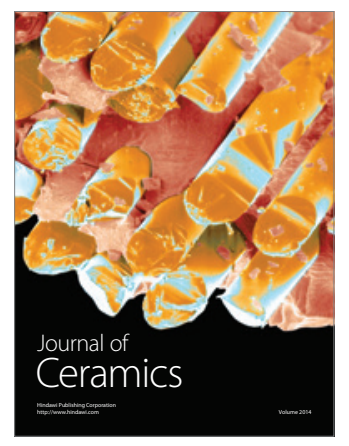

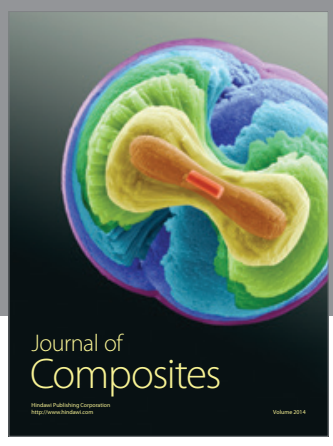
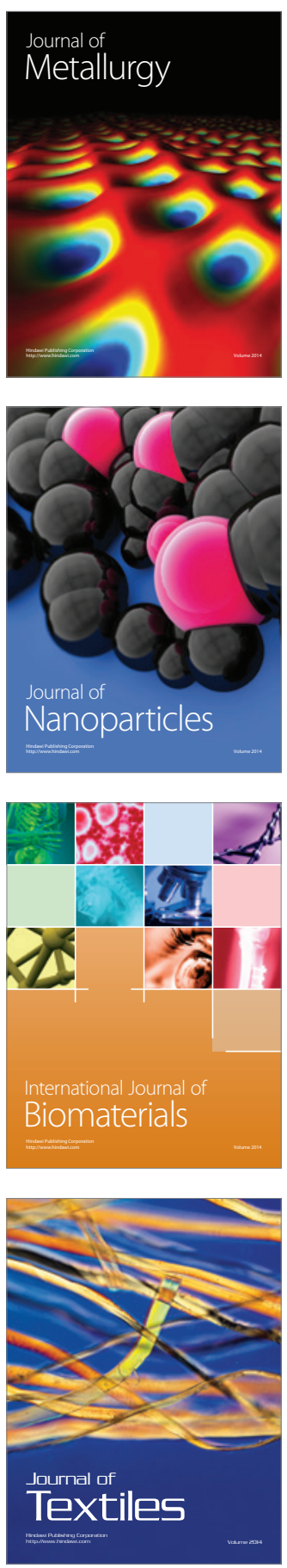